\title{
Effect of Pulse Energy on the Optical Properties of ZnS Thin Films Prepared Using Pulse Laser Technique
}

\author{
Khalid Mohammed Haroun ${ }^{1}$, Mohammed Nouman Mohammed Abdalla ${ }^{2}$, Sohad Saad Elwakeel ${ }^{2}$, \\ Yousif Hassan Alsheikh Abd Alraheim ${ }^{3}$
}

${ }^{1}$ Faculty of Radiobiological and Imaging Sciences, Alzaeim Al-Azhari University, Omdurman, Sudan

${ }^{2}$ Institute of Laser, Sudan University of Science \& Technology, Khartoum, Sudan

${ }^{3}$ Department of Applied Physics \& Mathematics, Omdurman Ahlia University, Omdurman, Sudan

Email address:

yousif.alsheikh@yahoo.com (Y. H. A. A. Alraheim)

\section{To cite this article:}

Khalid Mohammed Haroun, Mohammed Nouman Mohammed Abdalla, Sohad Saad Elwakeel, Yousif Hassan Alsheikh Abd Alraheim. Effect of Pulse Energy on the Optical Properties of ZnS Thin Films Prepared Using Pulse Laser Technique. American Journal of Modern Physics. Vol. 7, No. 5, 2018, pp. 173-179. doi: 10.11648/j.ajmp.20180705.11

Received: October 6, 2018; Accepted: October 22, 2018; Published: November 19, 2018

\begin{abstract}
In these work four samples of $\mathrm{ZnS}$ thin films deposited on glass substrate using pulse laser deposition method with different pulse energies, the effect of the laser pulse energy on the optical properties of the four ZnS thin films fabricated was studied. Q-Switched Nd: YAG laser with the fundamental wavelength $1064 \mathrm{~nm}$, laser Pulse energies of $(125,150,175$, and 200) mj with fixed number of pulses of 20 , and pulse repetition rate of $5 \mathrm{~Hz}$ were used. The target to the substrate distance and angle were kept fixed. The film thicknesses were measured using FESEM measurement tool. The thickness of the deposited $\mathrm{ZnS}$ thin films was found to be linearly dependent on the pulse energy used. The transmission spectra in the tested region (532 to 915$) \mathrm{nm}$ were found to be in the range from 0.41 to $0.59 \%$ depending on the $\mathrm{ZnS}$ thin film thickness, and for each $\mathrm{ZnS}$ thin film the transmission spectrum is unique. The refractive indices of all samples were determined; and for each sample and it were found to change with wavelength, the highest refractive index of 5.6 at $915 \mathrm{~nm}$ was obtained for the sample of the smallest thickness 0.49 microns. Transmission spectra, absorption coefficients and the refractive indices they were in good agreement with the literature.
\end{abstract}

Keywords: Laser Pulse Energy, Optical Properties, ZnS Thin Films, Pulse Laser Deposition (PLD)

\section{Introduction}

Zinc Sulphide (ZnS) is a wide gap and direct transition semiconductor that belong to group II-VI semiconductors [1,2]. $\mathrm{ZnS}$ thin films are believed to be one of the most promising materials for blue light emitting diodes, and in electroluminescent displays [3]. As a result, $\mathrm{ZnS}$ is an important material used as an antireflection coating in heterojunction solar cells [4]. And in Infrared windows [4]. There are many challenges to produce this material in thin film structure. There exist several methods to produce thin films from this material such as sol-gel, radiofrequency sputtering [5], pulse laser deposition [6], and so on.

Pulse laser deposition (PLD) has been successfully used to deposit an extraordinary wide range [7]. In thin films prepared by PLD technique a variety of deposition parameters corresponds to the laser properties such as laser pulse energy, pulse repetition rate, etc., in addition to the rest of the other deposition condition such as target - substrate distance and angle, substrate temperature, ambient gas and pressure are all fundamental deposition conditions that determines the properties of the fabricated films [8]. The PLD is widely used technique for the fabrication of thin films because of its numerous advantages such as its simplicity, etc [9]. The PLD technique enables the deposition of many complex materials over a wide range of background gas composition and pressures [8]. E. Ma'rquez et al. in (2014) fabricated $\mathrm{ZnS}$ thin films on glass substrate via thermal evaporation technique and studied their structural and optical properties [10]. In (2014) K. R. Murali deposited ZnS films by the pulse plating technique at different duty cycles in the range of $6-50 \%$ and at a constant current density of $10 \mathrm{~mA}$ $\mathrm{cm}^{-2}$ and he studied their Optical constants (refractive index, $\mathrm{n}$, 
extinction co-efficient, $\mathrm{k}$, dielectric constant) of the films in the wavelength range $300-1850 \mathrm{~nm}$ by using spectrophotometric measurement [1]. In this work four samples of $\mathrm{ZnS}$ thin film were fabricated using Q-switched Nd:YAG pulsed laser deposition with the wavelength of $1064 \mathrm{~nm}, 10$ pulses with pulse repetition rate of $2 \mathrm{~Hz}$, the target to substrate distance and angle were fixed to $2 \mathrm{~cm}$ and $45^{\circ}$, respectively. Varied pulse energy of (100,150, 180, 200 and 250) mj were used. The film thickness was measured by Field Emission scanning Electron Microscope (FESEM) measurement tool, and the transmission spectrum at certain wavelengths for each film was recorded. $\mathrm{ZnS}$ thin films transmission data and the measured film thicknesses were used to calculate their optical properties.

\section{Materials, Tools and Methods}

\subsection{Materials}

The material used in this work were $\mathrm{ZnS}$ of $99.9 \%$ purity and refractive index of 1.46 it was prepared in disc form by mixing 50:50 ratio of the $\mathrm{ZnS}$ powder and Potassium bromide (IR spectroscopy grade). The powder materials were pressed using hand pressing machine to form solid disks as target for ablation.

\subsection{Tools and Equipments}

Different tools and equipments were used to complete this work. They were described below with their specifications and needs:

\subsubsection{Pressing Machine}

A hand press machine manufactured by Shimadzu (Japan) was used in this work [11]. The machine is used to press the $\mathrm{ZnS}$ powder in disc form (after mixing with Potassium Bromide) for target ablation.

\subsubsection{Q-Switched Nd: YAG Laser}

Q-switched Nd: YAG model OW D1 was used to deposit

\section{ZnS thin film.}

\subsubsection{Scanning Electron Microscope (FESEM)}

Scanning electron microscopy type field emission model TESCAN MIRA3 was used to measure the films thickness. MIRA3 is a high performance SEM system which gives high resolution and low-noise imaging. MIRA3 offers all the advantages that come with the latest technologies and developments in SEM; delivering faster image acquisition, an ultra-fast scanning system, dynamic and static compensation and built-in scripting for user-defined applications $[6,11]$.

\subsubsection{Different Light Sources}

Different monochromatic light sources were used to record the transmission spectra for the fabricated films [12-14].

Table 1. The monochromatic light sources used in this work.

\begin{tabular}{ll}
\hline Light Source & Wavelength (nm) \\
\hline Diode Laser & 532 \\
He-Ne Laser & 632.8 \\
Diode laser & 660 \\
Omega XP Laser (red probe) & 675 \\
Omega XP Laser (IR probe) & 820 \\
Omega XP Laser (IR probe) & 915 \\
\hline
\end{tabular}

\subsubsection{The Photodetector}

A silicon pin photodiode was used in this work for detecting the transmitted intensity of each light source after passed through the fabricated $\mathrm{ZnS}$ films.

\subsection{The Method}

The procedure to fabricate $\mathrm{ZnS}$ thin films and study the effect of the pulse energy on their properties was done as follows:

The experimental setup used to produce $\mathrm{ZnS}$ thin films was arranged as shown in figure 1.

Different disks of $\mathrm{ZnS}$ (as targets) were prepared by the press machine.

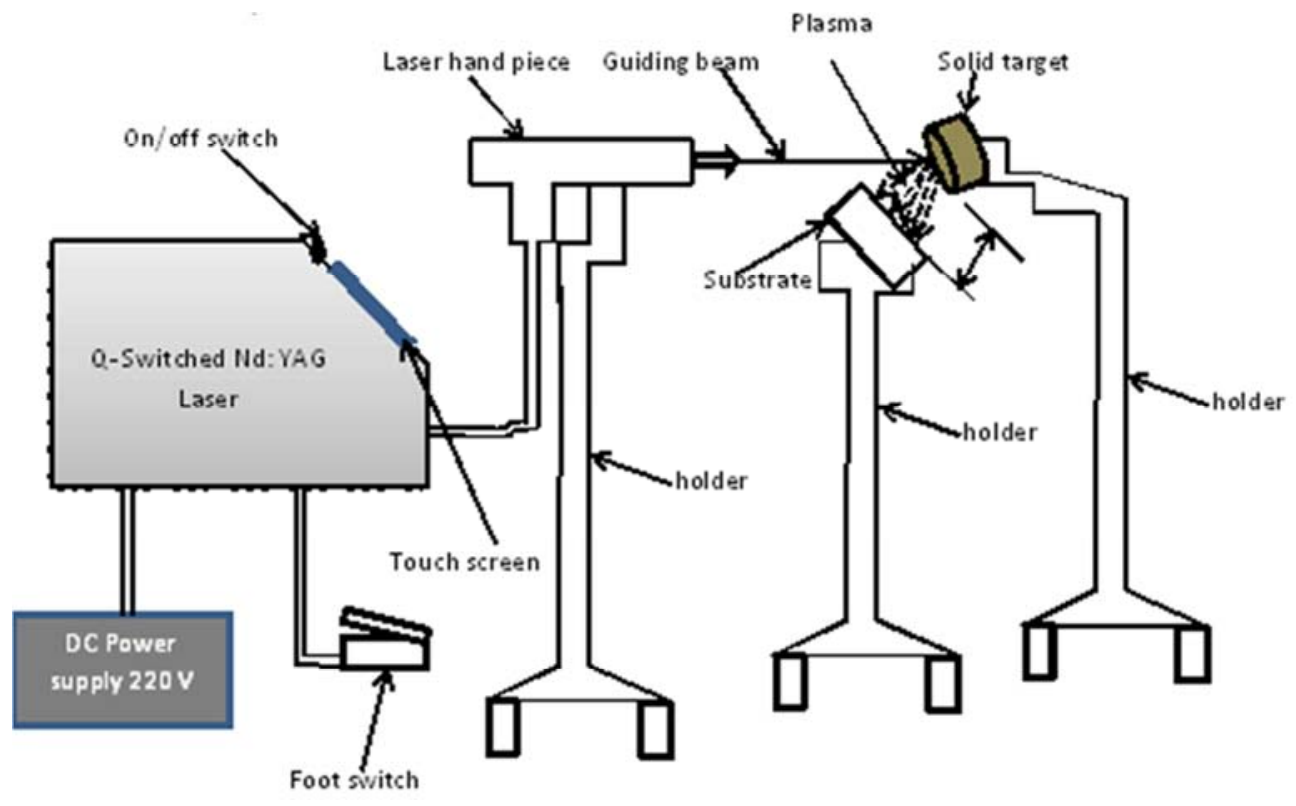

Figure 1. Schematic diagram of the experimental setup for fabrication of ZnS thin films. 
The distance and the angle between the target and the glass substrate were fixed to $3 \mathrm{~cm}$, and $45^{\circ}$, respectively.

The glass substrates were cut into the dimensions suitable for SEM imaging $2 \times 2 \mathrm{~cm}$, and then washed with distilled water and cleaned with alcohol.

The Q-Switched Nd: YAG laser machine was switched on and 10 pulses with energy of $100 \mathrm{mj}$ and a R. R. of $2 \mathrm{~Hz}$ was used to deposit $\mathrm{ZnS}$ thin film on the glass substrate.

The above step was repeated four times with varied pulse energy of $(125,150,175$ and 200) mj with fixed repetition rate and number of pulses.

The fabricated $\mathrm{ZnS}$ thin films were examined using FESEM to measure their thicknesses.

The relation between the laser pulse energy and the $\mathrm{ZnS}$ thin film thickness was plotted.

The transmission spectra of the fabricated $\mathrm{ZnS}$ films were recorded using different monochromatic light sources.

Thicknesses of the $\mathrm{ZnS}$ films and the transmission data were used to calculate the optical properties for each film.

The refractive index of each thin film was calculated using the measured reflectivity $\mathrm{R}$ and the glass refractive index $\mu_{\mathrm{s}}$ according to: $[15,16]$.

$$
\begin{aligned}
& \mu=\left(\frac{\mu_{s}[1+\sqrt{R}]}{1-\sqrt{R}}\right)^{\frac{1}{2}} \\
& \mu_{s}=\frac{1}{T_{s}}\left(\frac{1}{T_{s}^{2}}-1\right)^{\frac{1}{2}}
\end{aligned}
$$

where $\mathrm{T}_{\mathrm{s}}$ represents the transmission of the glass substrate.

The absorption coefficients were deduced from the measured value of reflectivity $R$, the transmittance $T$, refractive index $\mu_{\mathrm{s}}$, and thickness t according to $[15,16]$ :

$$
\alpha=\frac{1}{t} \mu \frac{(1-R)^{2}}{T}
$$

\section{Results and Discussion}

The results of this work are here were composed of two parts; the first part is: Pulse energy deposition parameter of the PLD on the thickness of the fabricated $\mathrm{ZnS}$ thin films and second part is: The effect of the pulse energy on the optical properties of the produced $\mathrm{ZnS}$ thin films.

\subsection{Pulse Energy Effect on the Thickness of the ZnS Thin Films}

Four $\mathrm{ZnS}$ thin films using 20 pulses with pulse repetition rate of $5 \mathrm{~Hz}$ and varying pulse energies of $(125,150,175$, and 200) mj were deposited on glass substrates. Then the four deposited $\mathrm{ZnS}$ thin film samples were imaged using FESEM machine and their thicknesses were measured and tabulated in table 2 with the corresponding pulse energy used. Figure 2 shows the morphology of the $\mathrm{ZnS}$ thin film deposited using laser pulse energy of $125 \mathrm{mj}$ and figure 3 shows the FESEM thickness measurement of the produced $\mathrm{ZnS}$ thin film.

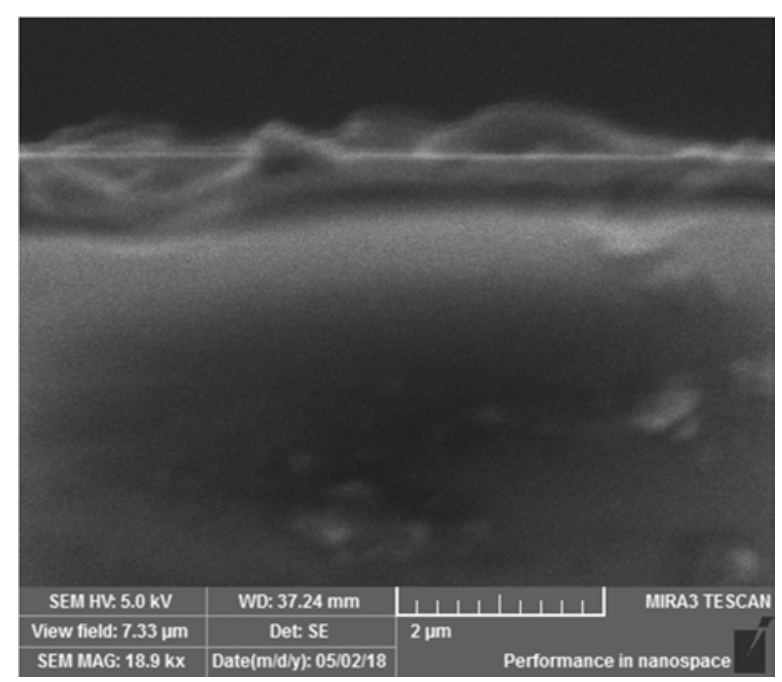

Figure 2. ZnS thin film deposited on glass substrate with laser energy of 125 mj and repetition rate of $5 \mathrm{~Hz}$.

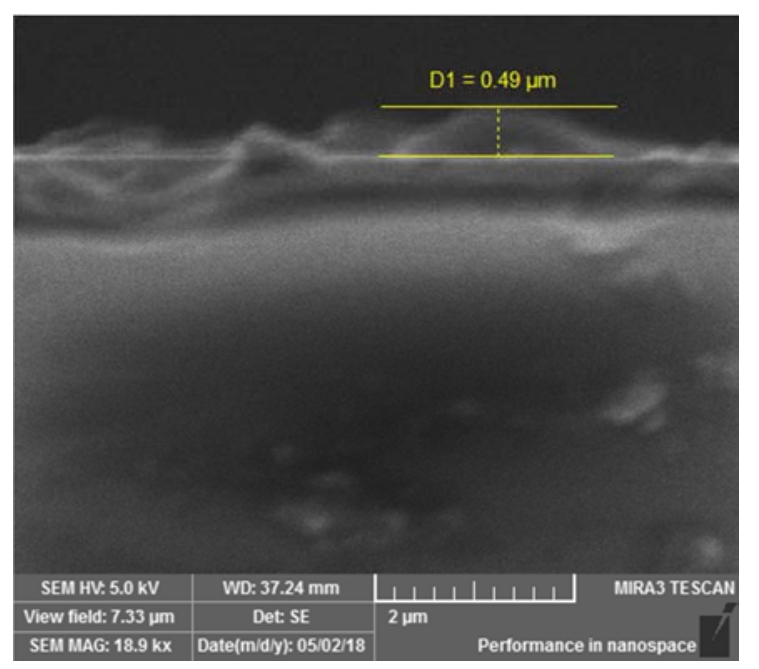

Figure 3. Thickness measurement of the ZnS thin film deposited on glass substrate with laser energy of $125 \mathrm{mj}$ and repetition rate of $5 \mathrm{~Hz}$.

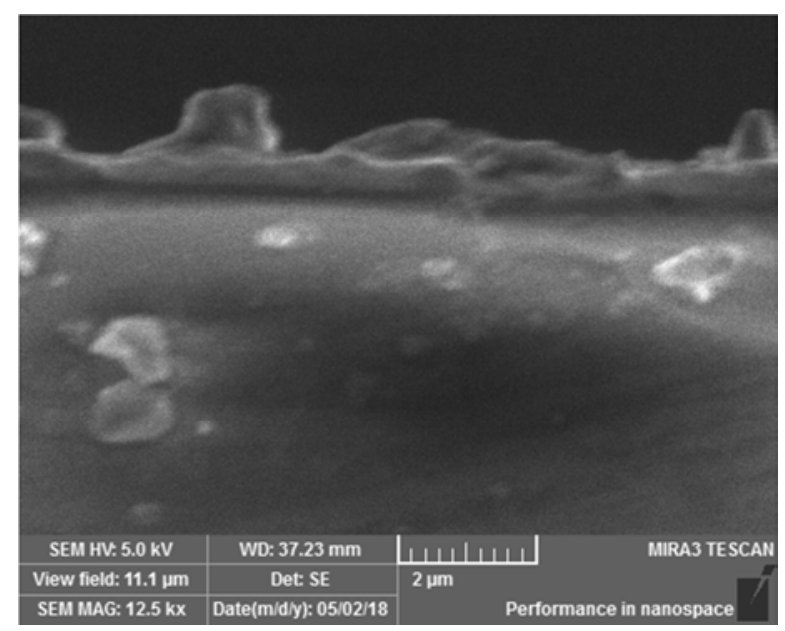

Figure 4. ZnS thin film deposited on glass substrate with laser energy of 200 mj and repetition rate of $5 \mathrm{~Hz}$.

The FESEM image together with the thickness measurement 
shown in figures 2 and 3, respectively, illustrate that the $\mathrm{ZnS}$ thin film has a thickness of $0.49 \mu \mathrm{m}$ and it's clear that the fabricated film is dense and has smooth film morphology.

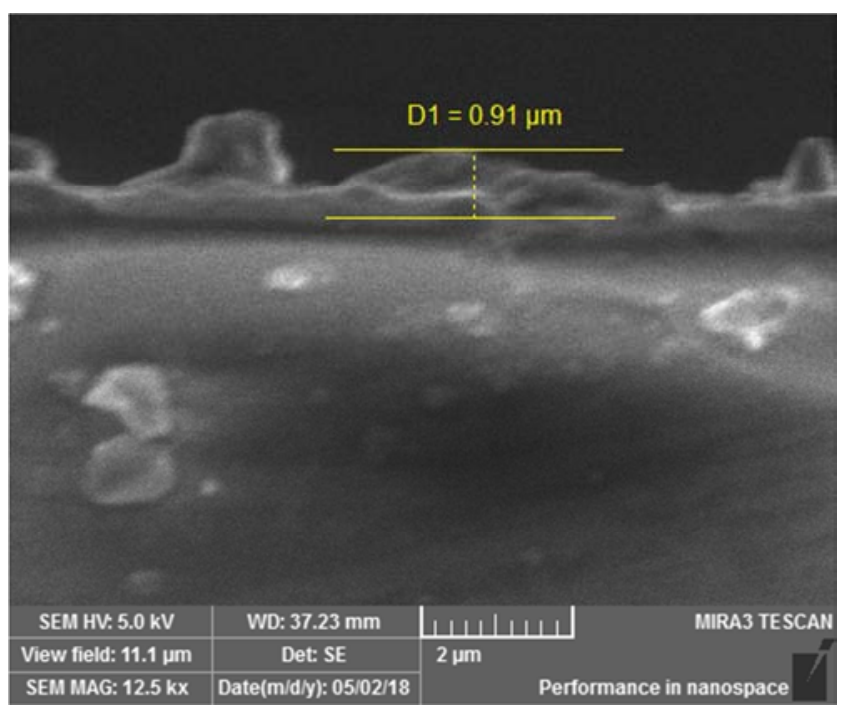

Figure 5. Thickness of ZnS thin film deposited on glass substrate with laser energy of $200 \mathrm{mj}$ and repetition rate of $5 \mathrm{~Hz}$.
Figure 4 shows the $\mathrm{ZnS}$ thin film that was achieved laser pulse energy of $200 \mathrm{mj}$, while the number of pulses, the pulse repetition rate and other deposition parameters were the same. The film thickness that results when the pulse energy was $200 \mathrm{mj}$ is shown in figure 5 .

Also figure 4 proved this film is dense and with nonsmooth morphology compared to the first film in figure 2 and this is due to the increasing the pulse energy.

Table 2. Thicknesses of the five fabricated ZnS thin films versus laser pulse energy.

\begin{tabular}{ll}
\hline Pulse energy in $(\mathbf{m j})$ with $\mathbf{R} . \mathbf{R}=\mathbf{2} \mathbf{~ H z}$ & $\mathbf{Z n S}$ thin film thickness in $(\boldsymbol{\mu m})$ \\
\hline 125 & 0.49 \\
150 & 0.52 \\
175 & 0.58 \\
200 & 0.91 \\
\hline
\end{tabular}

The results obtained showed that nanometric $\mathrm{ZnS}$ thin films of the order of 100 nanometers are easy fabricated using PLD.

The relation between the $\mathrm{ZnS}$ thin film thickness and the pulse energy is plotted together with its fitting in figure 6 , and it was showed that the thickness of the $\mathrm{ZnS}$ is linearly dependent on the laser pulse energy used for deposition.

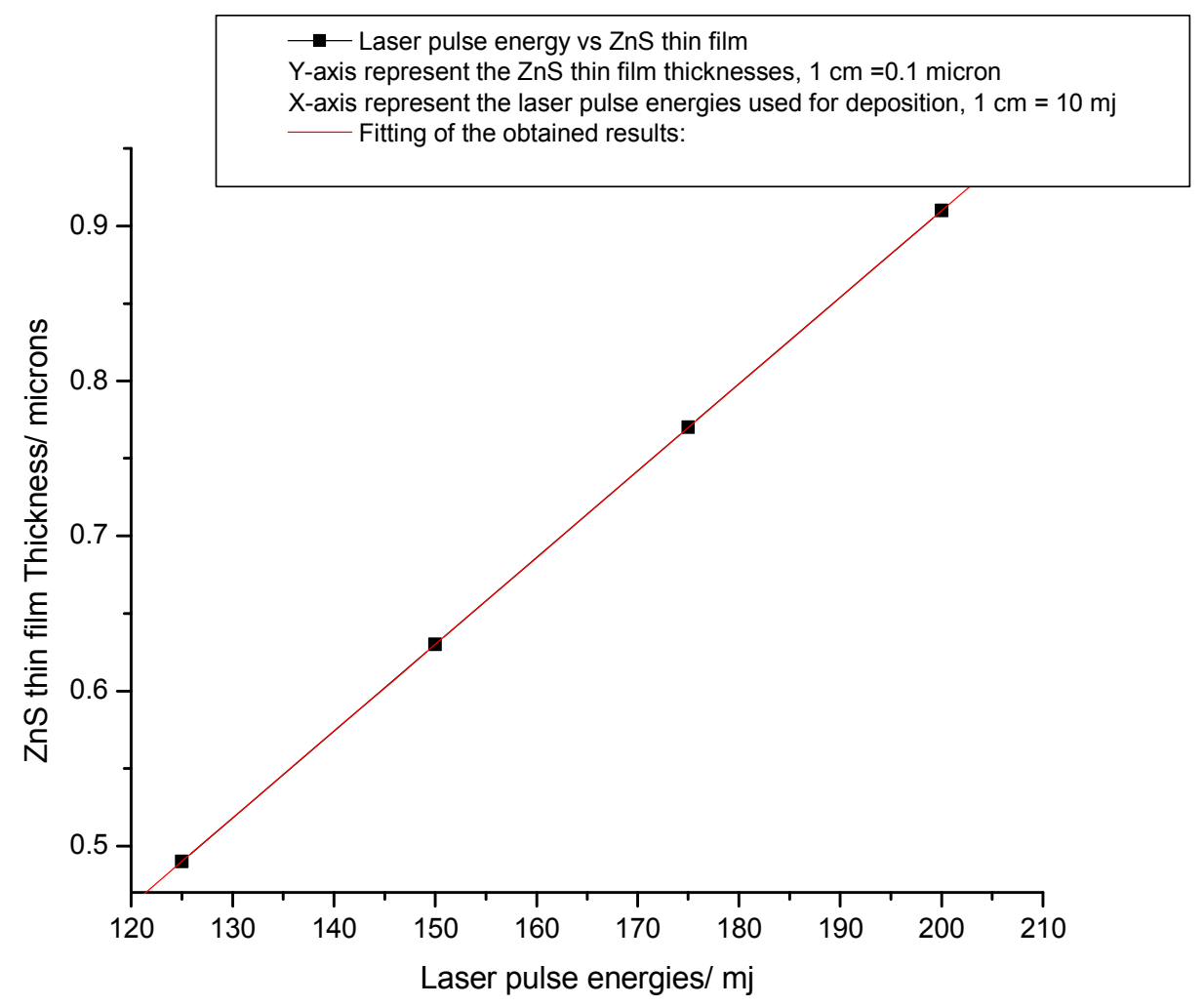

Figure 6. The ZnS thin film thicknesses versus pulse energy used for deposition.

From figure 6 it is clear that increasing the pulse energy results in an increment of the $\mathrm{ZnS}$ thin film thickness.

\subsection{Optical Properties of ZnS}

Then the transmission intensities of different monochromatic light sources were detected before and after deposition for the four $\mathrm{ZnS}$ thin film samples, and then the transmitted intensities were used to calculate the transmission percentage $\left(\mathrm{T} \%=\mathrm{I}_{\mathrm{t}} / \mathrm{I}_{\mathrm{o}}\right)$ at a certain monochromatic light wavelengths. The calculated values are plotted for the the four $\mathrm{ZnS}$ thin film samples $\mathrm{S}_{1}, \mathrm{~S}_{2}, \mathrm{~S}_{3}, \mathrm{~S}_{4}$ in figure 7 as a function of wavelengths. 


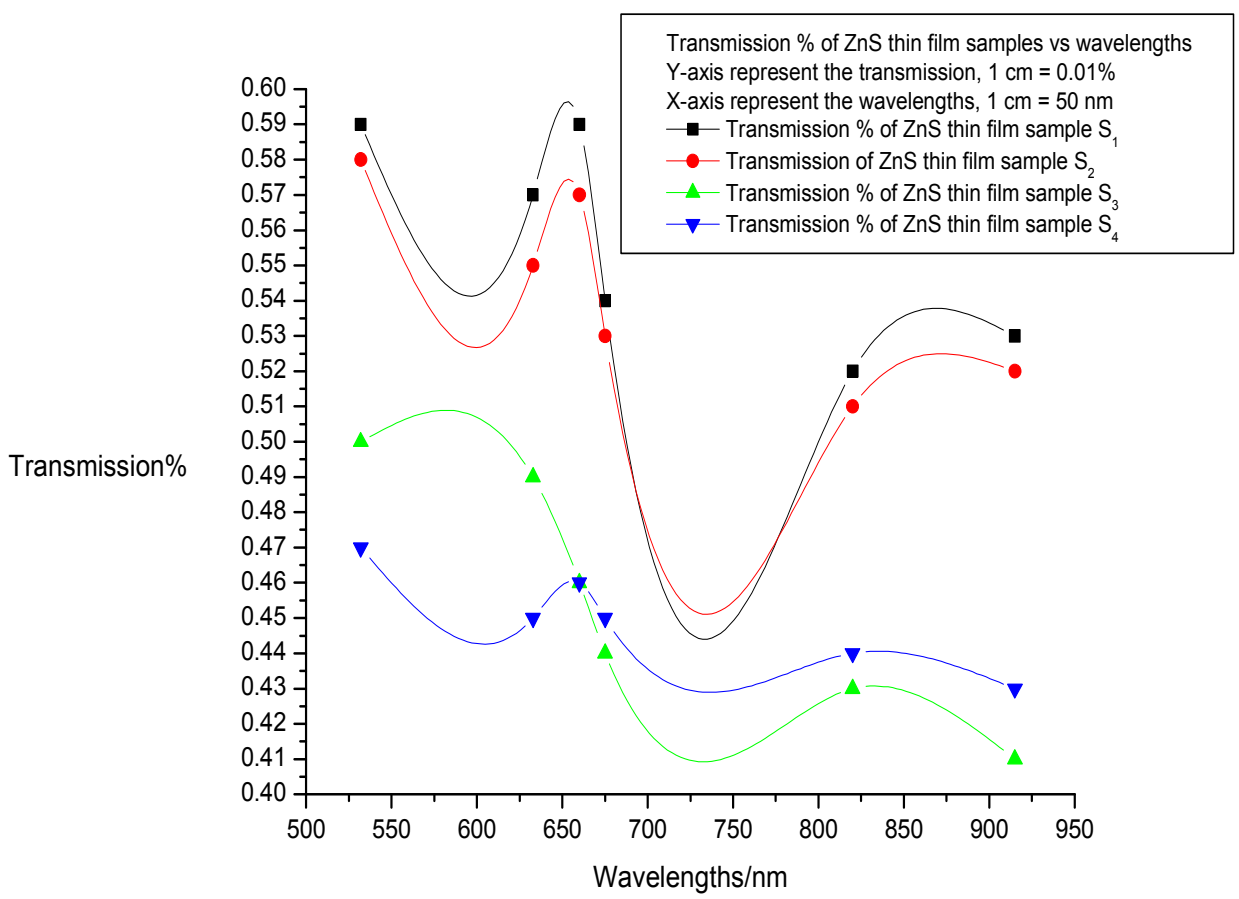

Figure 7. Transmission spectrum of the four ZnS thin film samples.

Figure 7 showed that the thickness of the thin film affected its transmission, comparing between the transmission of the four samples of $\mathrm{ZnS}$ thin films, samples $\mathrm{S}_{1}, \mathrm{~S}_{2}, \mathrm{~S}_{3}$, and $\mathrm{S}_{4}$ together with the results shown in figure 6 illustrates that the large thickness of the thin film and thus the higher the pulse energy used for deposition gives the lower transmission of the film.

The transmission spectra of all $\mathrm{ZnS}$ samples are in good agreement with the work of K. R. Murali in the region from $532 \mathrm{~nm}$ to $915 \mathrm{~nm}$.

The relation between the calculated refractive indices for the four samples of $\mathrm{ZnS}$ thin films deposited on glass substrates using pulsed laser technique with fixed pulse repetition rates and varied pulse energy and were done according to equation (1), and are plotted as a function of wavelengths as shown in figure 8 .

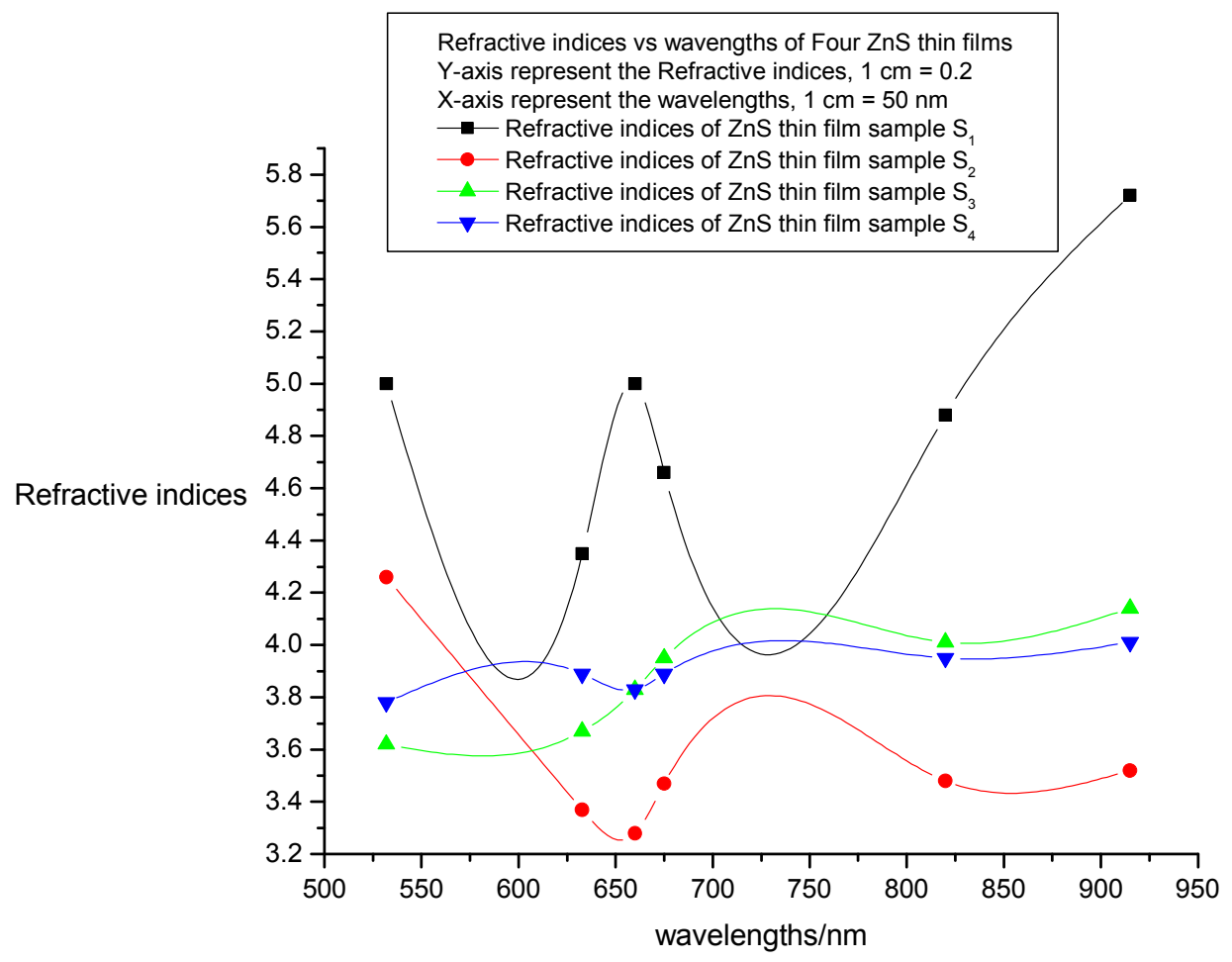

Figure 8. The refractive index of the four samples of $\mathrm{ZnS}$ thin films versus wavelengths. 
The refractive index of any material in thin film profile is usually deviates from that of the bulk of the same material [15]. This is due to the void fraction typical of the thin film microstructure. Figure 8 showed that $\mathrm{ZnS}$ thin film sample $\mathrm{S}_{1}$ which of smallest thickness among the other has highest refractive indices from 532 to $915 \mathrm{~nm}$ this result suggest using $\mathrm{ZnS}$ thin films in applications that require high index materials, and also the results in figure 8 proved that increasing the thickness of the thin film the refractive index decrease and when the thickness of the thin films is in the range of 1 micron or above the refractive index of the film deviates infinitesimally from that of the bulk material.

The absorption coefficients calculated for the four samples using equation (2) and were plotted versus wavelengths in figure 9 below:

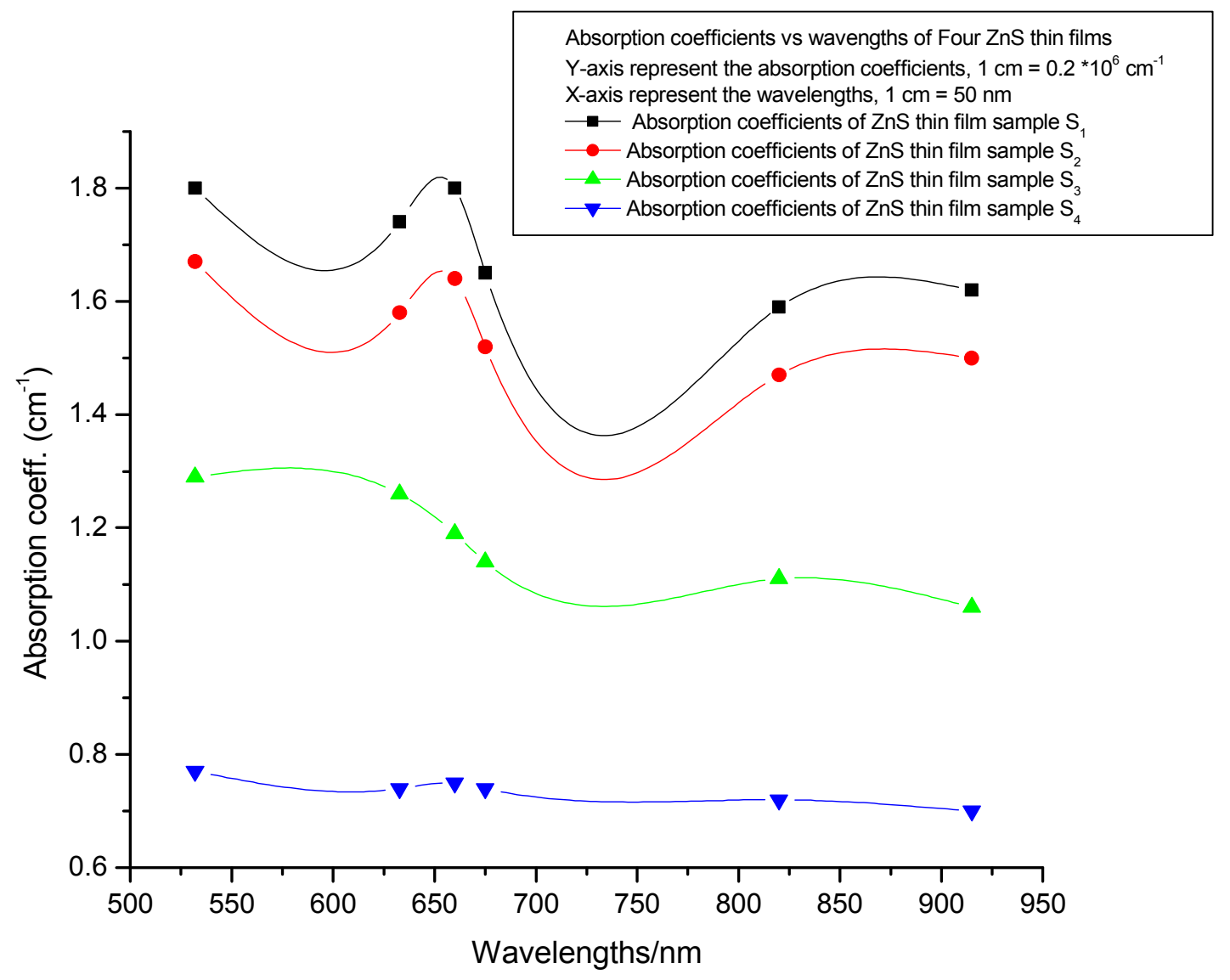

Figure 9. Absorption coefficients versus wavelengths for four samples of ZnS thin films.

Figures 8 and 9 support the idea of using such film as an optical filter or as a reflector in specific wavelengths deduced from the transmission spectrum, since the four samples has different absorption coefficients, the absorption coefficients of the $\mathrm{ZnS}$ thin films for all samples showed in figure 9 exhibits similar phenomenon with the wavelengths. The refractive indices and absorption coefficients of the pulsed laser deposited $\mathrm{ZnS}$ thin films varies with thickness and for each thickness the transmission is unique, therefore this two optical properties are functions in the film thickness and therefore on the repetition rate used for the deposition of such films. Optical measurement constitutes the most important means of determining the band structure of the materials. And the optical constants of thin films provide us with information concerning microscopic characteristics of the material and its determination is very important for using it in any of such devices $[6,8]$.

\section{Conclusions}

In this study, the effect of laser pulse energy on the thickness and optical properties of $\mathrm{ZnS}$ thin films deposited on glass substrate by pulse laser deposition method has been investigated. The $\mathrm{ZnS}$ thin deposited films thicknesses were measured using FESEM measurement tool. Transmission spectra of the deposited $\mathrm{ZnS}$ thin films at certain wavelengths were recorded. The measured $\mathrm{ZnS}$ thin film thicknesses and the transmission data were used to deduce the refractive indices and the absorption coefficients of each thin film. The transmission, refractive indices and absorption coefficients values obtained are in good agreement with the literature. In conclusion $\mathrm{ZnS}$ thin films deposited by pulse laser deposition method can be used to produce optical components in the range from visible to IR regions by controlling its thickness via controlling the laser pulse energy, the results also showed 
that the $\mathrm{ZnS}$ thin films are suitable for applications requiring high refractive index material.

\section{References}

[1] K. R. Murali (2014), Optical properties of pulse electrodeposited $\mathrm{ZnS}$ films, IOSR Journal of Applied Physics (IOSR-JAP), Volume 6, Issue 3 Ver. II, pp. 9-14.

[2] R. Maity and K. K. Chattopadhyay (2004), Synthesis and optical characterization of $\mathrm{ZnS}$ and $\mathrm{ZnS}$ : Mn nanocrystalline thin films by chemical route, Nanotechnology, volume 15, issue 7, pp. 812-816.

[3] Mahdi Hasan Suhail and Raoof Ali Ahmed (2014), Structural, optical and electrical properties of doped copper $\mathrm{ZnS}$ thin films prepared by chemical spray pyrolysis technique, Advances in Applied Science Research, volume 5, issue 5, pp. 139-147.

[4] J. Mohamed (2002), the study of structural and optical properties for thin film ( $\mathrm{PbS})$ and $\mathrm{ZnS},(M$. Sc. thesis), University of Tikrit, Iraq.

[5] Kumar V, Saroja M., Venkatachalam M, and Shankar S (2015), Synthesis and characterization of $\mathrm{ZnS}$ thin films by Sol-gel and dip spin coating methods, International of Recent Scientific Research, volume 6, issue 11, pp. 7377-7379.

[6] Nafie A. Almuslet, Kh. M. Haroun, and Yousif H. Alsheikh (2018), Pulse Energy Effect on the Optical Properties of Pulse Laser Deposited $\mathrm{SiO}_{2}$ Thin Films, International Journal of Trend in Scientific Research and Development (IJTSRD), Volume - 2, Issue - 6, Sep - Oct 2018, pp. 47-54.

[7] Mohammed A. Hameed (2015), Physical Properties of
Nanostructured Silicon Dioxide Prepared by Pulsed-Laser Deposition, Journal of Physical Vapor Deposition Science and Technology, Volume 9, issue 10, pp. 451-454.

[8] Nafie A. Almuslet and Yousif H. Alsheikh (2018), Effect of Pulse Repetition Rate on the Properties of Pulse Laser Deposited $\mathrm{SiO}_{2}$ Thin Films, Presented at ICCCEEE18 (12-14 August 2018), University of Gazira, Sudan.

[9] E. Ma'rquez, E. R. Shaaban, and A. M. Abousehly, Structural and optical properties of $\mathrm{ZnS}$ thin films, International Journal of New Horizons in Physics, volume 1, issue 1, pp. 17-24.

[10] Shimadzu hand press machine, product information (P/N 20064174), Shimadzu Corporation, Japan, 2013

[11] Microscopy - MATERIAL SCIENCE - Electron Microscopy TESCAN, MIRA3, [online] available from https://www.wirsam.com/product/mira-3, visited, 16/2/2018.

[12] Phywe, Helium Neon Laser Products 8181-93 Info. 2007, https://www.phywe.de/en/geraetehierarchie/physics/modernph ysics/quantum-physics/08181-93.

[13] Oriental Wison, 1320/1064/532 nm Q-switched Nd: YAG laser, Model Name OW-D1, product datasheet, Beijing Oriental Wison Mechanical \& Electronic Co., Ltd, 2017, China.

[14] Biotechhealth, Omega XP laser system and product datasheet, $2011 \mathrm{http} / / \mathrm{www}$. biotechhealth.co.uk.

[15] Nafie A. Almuslet and Yousif H. Alsheikh (2015), Investigation of the Optical Properties of Liquid Deposition $\mathrm{CuSO}_{4}$ Thin Film, IJSRST, Volume 1, issue 5, pp. 132-136.

[16] M. T. Hussein, E. M. Nasir, and A. H. Al-Aarajiy (2012), Study on the UV-Visible of Ni-Phthalocyanine thin film Optical Properties, Int. J. Thin Film Sci. Tec. 1, No. 2, 71-76. 